\title{
High Accuracy Numerical Methods for Thermally Perfect Gas Flows with Chemistry
}

\author{
Ronald P. Fedkiw, Barry Merriman, and Stanley Osher ${ }^{1}$ \\ Department of Mathematics, University of California, 405 Hilgard Avenue, Los Angeles, California 90095-1555
}

Received July 25, 1996

\begin{abstract}
The compressible Navier Stokes equations can be extended to model multi-species, chemically reacting gas flows. The result is a large system of convection-diffusion equations with stiff source terms. In this paper we develop the framework needed to apply modern high accuracy numerical methods from computational gas dynamics to this extended system. We also present representative computational results using one such method. The framework developed here is useful for many modern numerical schemes. We first present an enthalpy based form of the equations that is well suited both for physical modeling and for numerical implementation. We show how to treat the stiff reactions via time splitting, and in particular how to increase accuracy by avoidng the common practice of approximating the temperature. We derive simple, exact formulas for the characteristics of the convective part of the equations, which are essential for application of all characteristic-based schemes. We also show that the common practice of using approximate analytical expressions for the characteristics can potentially produce spurious oscillations in computations.

We implement these developments with a particular high accuracy characteristic-based method, the finite difference ENO space discretization with the 3rd order TVD Runge-Kutta time discretization, combined with the second order accurate Strang time splitting of the reaction terms. We illustrate the capabilities of this approach with calculations of a 1-D reacting shock tube and a 2-D combustor. (c) 1997 Academic Press
\end{abstract}

\section{INTRODUCTION}

Chemically reacting, high speed gas flows arise in a variety of combustion problems, such as the fueling of a scramjet engine or the incineration of waste in a dump combustor. The combination of energetic chemical reactions and compressible gas dynamics yields the unique phenomena of detonation and deflagration. The basic properties of these effects can be understood via the ChapmanJouget theory.

For theoretical modeling or numerical simulation of such flows, the compressible Navier Stokes equations can be extended to include multiple gas species and the appropriate chemical reactions. The standard approach is to con-

\footnotetext{
${ }^{1}$ Research supported in part by ARPA URI-ONR-N00014-92-J-1890, NSF DMS 94-04942, and ARO DAAL03-91-G-0162.
}

sider the total mixture as a single compressible fluid, with the species-averaged density, momentum, and energy evolving according to the corresponding conservation laws. In addition, the mass fraction of each species evolves according to a separate continuity equation. These continuity equations are strongly coupled through the chemical reactions, and they also couple strongly to the equations for the mixture via the effect of reactions on temperature and pressure.

Since chemical reactions can cause large localized temperature variations during combustion, it is important to accurately include the temperature dependencies in the equations of state used for the gas species. The most tractable model that includes a realistic temperature dependence is that of a thermally perfect gas, for which the heat capacities can be general functions of temperature. In practice these functions are based on experimental data and they differ significantly from the ideal gas law at the higher temperatures encountered during combustion.

By considering the mathematical and physical character of the problem, we can pose some general requirements for suitable numerical methods. The resulting model equations form a large system of nonlinear conservation laws with both first and second order derivative terms (from convective and diffusive transport) and zeroth order source terms (from reactions). Because the diffusive terms are weak, we expect that the spatial transport terms will result in the development of steep fronts. Because the reactions proceed rapidly once they are triggered, we expect that the source terms will be stiff in time. Thus any numerical approach must effectively handle stiff time integration and steep spatial fronts.

Since the stiff source terms require specialized and costly time integration, it is most practical to use a time splitting to isolate their treatment from the rest of the problem. To handle the steep spatial fronts, it is natural to apply modern shock-capturing numerical methods for the convective part of the conservation laws. These methods typically require complete analytic expressions for the characteristic data, i.e., the eigenvalues and eigenvectors of the linearized convective flux matrix. 
Based on these general considerations, we expect many numerical approaches will have a common need for a proper time split formulation and analytic expressions for the characteristic data. Obtaining both these things would seem routine, but in fact the complexity of the equations makes both potentially difficult and has led to the use of a variety of simplifying procedures which may cause unanticipated errors in the computations, as some of our examples will illustrate. Our primary goal here is to show that, with the equations properly formulated, both the time splitting and characteristic data can be obtained without simplifying assumptions in an unambiguous and practically useful form. We also show that with these in hand, modern characteristic based methods do an excellent job of capturing the phenomena present in chemically reacting gas flows.

We develop our framework as follows: first, we present an enthalpy based formulation of the governing equations, i.e., the energy equation for the mixture is written in terms of the enthalpy. Various other equivalent forms are possible, such as using temperature or internal energy as the explicit variable, but the enthalpy formulation is advantageous for two reasons: it is convenient for physical modeling, and it results in a system for which the characteristics can be determined analytically in a compact and relatively simple form.

Then, we show how to apply time splitting to these equations in order to isolate the time evolution of the stiff reaction terms. In the previous work there has been some ambiguity regarding what terms should be held constant in the reaction portion of this time split evolution. For example, it has been a common practice to freeze the temperature during this step, but this is not a true time splitting of the model equations. Given the strong temperature dependence of the reaction rates, this is also a physically questionable practice. Others have considered adding an additional ODE for the simultaneous evolution of temperature with the reaction ODEs, but this approach adds unnecessary complication and also requires a decision about which thermodynamic quantities are being held constant during the step. In contrast, we show that a proper time splitting of the stiff reaction terms unambiguously requires that certain thermodynamic quantities (not temperature) be held constant during the solution of the reaction ODEs, and further we show that a simple scalar root finding procedure, such as Newton's method, is all that is required to implement this proper time splitting.

Next, we derive simple expressions for the characteristic data, i.e., Jacobian matrix of the convective fluxes, and the associated eigenvalues and eigenvectors. These are the primary ingredients needed to apply a variety of modern high accuracy characteristic based methods developed for gas dynamics.

Finally, we illustrate the capabilities of this numerical approach. We implement this framework using thermodynamic and chemistry data tables from CHEMKIN, and numerics consisting of second order Strang time splitting with a stiff ODE integrator (LSODE) for the reaction equations, 3rd order TVD Runge-Kutta time integration for the convection-diffusion terms, central differencing for the diffusive terms, and 3rd order finite difference ENO for the characteristic based discretization of the convection terms. We apply this to a one-dimensional Sod shock tube in the presence of combustion reactions, and to a twodimensional model of a toxic waste combustor, and discuss the results.

\section{MODEL EQUATIONS}

\subsection{Multiple Species}

The 2-D Euler equations can be modified to account for compressible flows with more than one species. The 2-D Euler equations for multi-species flow are

$$
\begin{aligned}
& \mathbf{U}_{t}+[\mathbf{F}(\mathbf{U})]_{x}+[\mathbf{G}(\mathbf{U})]_{y}=0 \\
& \mathbf{U}=\left(\begin{array}{c}
\rho \\
\rho u \\
\rho v \\
E \\
\rho Y_{1} \\
\vdots \\
\rho Y_{N-1}
\end{array}\right), \quad \mathbf{F}(\mathbf{U})=\left(\begin{array}{c}
\rho u \\
\rho u^{2}+p \\
\rho u v \\
(E+p) u \\
\rho u Y_{1} \\
\vdots \\
\rho u Y_{N-1}
\end{array}\right), \\
& \mathbf{G}(\mathbf{U})=\left(\begin{array}{c}
\rho v \\
\rho u v \\
\rho v^{2}+p \\
(E+p) v \\
\rho v Y_{1} \\
\vdots \\
\rho v Y_{N-1}
\end{array}\right) \\
& E=-p+\frac{\rho\left(u^{2}+v^{2}\right)}{2}+\rho h,
\end{aligned}
$$

where $E$ is the energy per unit volume, $h$ is enthalpy per unit mass, $N$ is the number of species being considered, and $Y_{i}$ is the mass fraction of the $i$ th species [16]. Note that $Y_{N}=1-\sum_{i=1}^{N-1} Y_{i}$.

\subsubsection{Energy and Enthalpy}

The total energy per unit volume is designated by $E$. We can write 


$$
E=\rho e+\frac{\rho\left(u^{2}+v^{2}\right)}{2},
$$

where $e$ is the internal energy per unit mass. We write the enthalpy per unit mass as

$$
h=e+\frac{p}{\rho}=\sum_{i=1}^{N} Y_{i} e_{i}+\frac{\sum_{i=1}^{N} p_{i}}{\rho}=\sum_{i=1}^{N} Y_{i}\left(e_{i}+\frac{p_{i}}{\rho Y_{i}}\right)=\sum_{i=1}^{N} Y_{i} h_{i}
$$

where $e_{i}, p_{i}$, and $h_{i}$ are the internal energy, partial pressure, and the enthalpy per unit mass of the $i$ th gas, respectively. We can likewise define

$$
H=\frac{E}{\rho}+\frac{p}{\rho}=\frac{E+p}{\rho}=h+\frac{u^{2}+v^{2}}{2}
$$

as the total enthalpy of the mixture. Using Eq. (5) to eliminate $e$ in Eq. (4) we can write

$$
E=-p+\frac{\rho\left(u^{2}+v^{2}\right)}{2}+\rho h
$$

as our energy equation.

In a perfect gas, the internal energy, enthalpy, and specific heats are functions of the temperature only. In this case we can write

$$
\begin{aligned}
h_{i} & =h_{i}(T) & e_{i} & =e_{i}(T) \\
c_{p i} & =c_{p i}(T) & c_{v i} & =c_{v i}(T)
\end{aligned}
$$

for a perfect gas, where $c_{p i}$ is the specific heat at constant pressure of the $i$ th species, and $c_{v i}$ is the specific heat at constant volume of the $i$ th species. Two other relationships which hold for a perfect gas,

$$
d h_{i}(T)=c_{p i}(T) d T \quad d e_{i}(T)=c_{v i}(T) d T
$$

will be very useful [1].

We can integrate both sides of the first equation in (10) to get

$$
h_{i}(T)=h_{i}(0)+\int_{0}^{T} c_{p i}(s) d s
$$

We can further classify perfect gases into two categories. A thermally perfect gas is one in which the specific heats are non-constant functions of temperature [1]. A calorically perfect gas is one in which the specific heats are constant [1]. Thus, Eq. (11) can be simplified, in the case of a calorically perfect species,

$$
h_{i}(T)=h_{i}^{f}+c_{p i} T
$$

where $h_{i}^{f}=h_{i}(0)$ is the enthalpy per unit mass at $0 \mathrm{~K}$ for the $i$ th species. This is also sometimes called the heat of formation. The heat of formation for a gas is a constant and can be found in the JANAF Thermochemical Tables [13]. We can rewrite Eq. (11) for a thermally perfect species,

$$
h_{i}(T)=h_{i}^{f}+\int_{0}^{T} c_{p i}(s) d s
$$

using the heat of formation.

The final result from Eq. (5),

$$
h=\sum_{i=1}^{N} Y_{i} h_{i}
$$

defines the enthalpy for a mixture of gases. Each thermally perfect species utilizes Eq. (13), while each calorically perfect species utilizes Eq. (12). The enthalpy formulation of the energy equation results in a convenient form for physical modeling, because the enthalpy is tabulated as a function of temperature for many gases. Also, this form allows us to readily model a gas as thermally perfect in one temperature regime and calorically perfect in another. Such flexibility can be used to investigate the effects of the thermally and calorically perfect assumptions.

Two common examples are worth noting. If all the species are thermally perfect,

$$
h=\sum_{i=1}^{N} Y_{i} h_{i}^{f}+\int_{0}^{T} \sum_{i=1}^{N} Y_{i} c_{p, i}(s) d s=\sum_{i=1}^{N} Y_{i} h_{i}^{f}+\int_{0}^{T} c_{p}(s) d s,
$$

where $c_{p}$ is the total specific heat at constant pressure of the mixture. If all the species are calorically perfect,

$$
h=\sum_{i=1}^{N} Y_{i} h_{i}^{f}+c_{p} T
$$

\subsubsection{Equation of State}

For a mixture of perfect gases, each gas has partial pressure,

$$
p_{i}=\rho Y_{i} R_{i} T
$$

where the specific gas constant $R_{i}$ for each species is

$$
R_{i}=\frac{R_{u}}{W_{i}}
$$

where $R_{u}=8314 \mathrm{~J} /(\mathrm{kmol} \mathrm{K})$ is the universal gas constant, 
and $W_{i}$ is the molecular weight of the $i$ th species [13]. Next we define $R$ as

$$
R=\sum_{i=1}^{N} Y_{i} R_{i}
$$

and we can write the equation of state for multi-species flow

$$
p=\sum_{i=1}^{N} p_{i}=\sum_{i=1}^{N} \rho Y_{i} R_{i} T=\rho\left(\sum_{i=1}^{N} Y_{i} R_{i}\right) T=\rho R T
$$

which is valid for mixtures of calorically perfect and thermally perfect gases [1].

\subsubsection{Specific Heats and Gamma}

We define gamma

$$
\gamma=\frac{c_{p}}{c_{v}}
$$

as the ratio of specific heats [2]. For a calorically perfect gas, $\gamma$ is constant. It is not unreasonable to assume that air at standard conditions is calorically perfect with $\gamma=$ 1.4. For a thermally perfect gas, $\gamma=\gamma(T)$ is a function of the temperature.

Another useful equation

$$
c_{p}-c_{v}=R
$$

can be used with Eq. (21) to get

$$
\gamma=\frac{c_{p}}{c_{p}-R}
$$

which is also valid for both calorically perfect and thermally perfect gases [2].

The specific heat and molecular weight of the mixture are given by

$$
\begin{aligned}
& c_{p}=\sum_{i=1}^{N} Y_{i} c_{p i} \\
& W=\frac{1}{\sum_{i=1}^{N}\left(Y_{i} / W_{i}\right)},
\end{aligned}
$$

where the unexpected form of Eq. (25) is explained in [8]. Gamma for the mixture is given by

$$
\gamma=\frac{c_{p}}{c_{p}-R_{u} / W}
$$

with $c_{p}$ and $W$ defined in Eqs. (24) and (25), respectively $[16,15]$. Note that for a mixture of calorically perfect gases, $\gamma=\gamma\left(Y_{i}\right)$ is a function of the mass fractions. For a mixture of thermally perfect gases, $\gamma=\gamma\left(Y_{i}, T\right)$ is a function of both the mass fractions and the temperature.

\subsection{Diffusive Transport and Chemical Reactions}

The 2-D Euler equations for multi-species flow can be further modified to account for viscosity, heat conduction, mass diffusion, and chemical reactions. The modified equations are the 2-D Navier Stokes equations for multi-species flow with chemical reactions

$$
\begin{aligned}
\mathbf{U}_{t}+[\mathbf{F}(\mathbf{U})]_{x}+[\mathbf{G}(\mathbf{U})]_{y} & =\left[\mathbf{F}_{v}(\mathbf{U})\right]_{x}+\left[\mathbf{G}_{v}(\mathbf{U})\right]_{y}+\mathbf{S} \\
E & =-p+\frac{\rho\left(u^{2}+v^{2}\right)}{2}+\rho h,
\end{aligned}
$$

where $\mathbf{U}, \mathbf{F}(\mathbf{U})$, and $\mathbf{G}(\mathbf{U})$ are given by Eq. (2), and the source term $\mathbf{S}$ is defined as

$$
\mathbf{S}=\left(\begin{array}{c}
0 \\
0 \\
0 \\
0 \\
\dot{\omega}_{1}\left(T, p, Y_{1}, Y_{2}, \ldots, Y_{N-1}\right. \\
\vdots \\
\dot{\omega}_{N-1}\left(T, p, Y_{1}, Y_{2}, \ldots, Y_{N-1}\right)
\end{array}\right)
$$

where $\dot{\omega}_{i}$ is the mass production rate of the $i$ th species [16]. Also $\mathbf{F}_{v}(\mathbf{U})$ and $\mathbf{G}_{v}(\mathbf{U})$ are given by

$$
\begin{gathered}
F_{v}(U)=\left(\begin{array}{c}
0 \\
\tau_{11} \\
\tau_{12} \\
u \tau_{11}+v \tau_{12}+Q_{1} \\
\rho D_{1, m}\left(Y_{1}\right)_{x} \\
\vdots \\
\rho D_{N-1, m}\left(Y_{N-1}\right)_{x}
\end{array}\right), \\
G_{v}(U)=\left(\begin{array}{c}
\tau_{12} \\
\tau_{22} \\
u \tau_{12}+v \tau_{22}+Q_{2} \\
\rho D_{1, m}\left(Y_{1}\right)_{y} \\
\vdots \\
\rho D_{N-1, m}\left(Y_{N-1}\right)_{y}
\end{array}\right)
\end{gathered}
$$




$$
\begin{aligned}
\tau_{11} & =\frac{2}{3} \mu\left(2 u_{x}-v_{y}\right), \quad \tau_{12}=\mu\left(u_{y}+v_{x}\right), \\
\tau_{22} & =\frac{2}{3} \mu\left(2 v_{y}-u_{x}\right) \\
Q_{1} & =k T_{x}+\rho \sum_{i=1}^{N} h_{i} D_{i, m}\left(Y_{1}\right)_{x} \\
Q_{2} & =k T_{y}+\rho \sum_{i=1}^{N} h_{i} D_{i, m}\left(Y_{i}\right)_{y}
\end{aligned}
$$

where $\mu$ is the mixture viscosity, $k$ is the mixture thermal conductivity, and $D_{i, m}$ is the mass diffusivity of species $i$ into the mixture [1]. For the detailed forms of these terms, see [8].

\section{NUMERICAL METHODS}

\subsection{Numerical Approach}

Consider the 2-D Navier Stokes equations for multispecies flow with chemical reactions given by Eq. (27). We solve these equations using a time splitting scheme. We will use Strang Splitting [14], which is 2nd order accurate, to incorporate the chemistry. We do not use splitting for the fluid dynamic equations. The method consists of solving two separate ordinary differential equations which have right hand sides adding to the right hand side of Eq. (27),

$$
\begin{aligned}
\mathbf{U}_{t}+[\mathbf{F}(\mathbf{U})]_{x}+[\mathbf{G}(\mathbf{U})]_{y} & =\left[\mathbf{F}_{v}(\mathbf{U})\right]_{x}+\left[\mathbf{G}_{v}(\mathbf{U})\right]_{y} \\
\mathbf{U}_{t} & =\mathbf{S},
\end{aligned}
$$

where the first of these is the 2-D Navier Stokes equations for multi-species flow without chemical reactions, and the second is a purely reacting equation. In one step we allow a non-reacting fluid to convect and diffuse. In the other step we allow a motionless fluid to react.

The success of Strang splitting depends on the operators being split apart and on the smoothness of the underlying solution. As an extreme example, one cannot split apart the two spatial convection terms of the discretized 2-D Euler equations, because the truncation error due to noncommutativity of operators causes a "blow-up" of the solution. In our case the splitting works well, since the source terms are not overly stiff. If we had a much more stiff source term, the time step for an accurate time splitting would become overly restrictive. In that case, to use a practical time step and prevent unresolved (in space and time) reaction fronts from propagating at incorrect speeds, we would need to use a temperature minimizing procedure such as that described in $[5,6,16,11]$.

Consider Eq. (33). We evaluate the hyperbolic terms, $[\mathbf{F}(\mathbf{U})]_{x}$ and $[\mathbf{G}(\mathbf{U})]_{y}$, with the ENO method [12]. The parabolic terms, $\left[\mathbf{F}_{v}(\mathbf{U})\right]_{x}$ and $\left[\mathbf{G}_{v}(\mathbf{U})\right]_{y}$, are evaluated with standard conservative central differencing. Once all terms have been computed, we update in time with TVD Runge-
Kutta methods [12,9]. The viscosity, $\mu_{i}=\mu_{i}(T)$, and thermal conductivity, $k_{i}=k_{i}(T)$, of each species depend on the temperature. The binary diffusion coefficients, $D_{j i}=$ $D_{j i}(T, p)$, are functions of the temperature and pressure. All of these can be accurately evaluated with a chemical kinetics package, such as CHEMKIN [10].

Consider Eq. 34. The first four equations of this system imply that $\rho_{t}=(\rho u)_{t}=(\rho v)_{t}=E_{t}=0$. Thus, $\rho, u, v$, and $E$ are constants. Using the fact that $\rho$ is constant along with Eq. (29), we see that solving Eq. (34) reduces to solving the following system of ordinary differential equations,

$$
\left(\begin{array}{c}
Y_{1} \\
Y_{2} \\
\vdots \\
Y_{N-1}
\end{array}\right)_{t}\left(\begin{array}{c}
\frac{\dot{\omega}_{1}\left(T, \rho, Y_{1}, Y_{2}, \ldots, Y_{N-1}\right)}{\rho} \\
\frac{\dot{\omega}_{2}\left(T, \rho, Y_{1}, Y_{2}, \ldots, Y_{N-1}\right)}{\rho} \\
\vdots \\
\frac{\dot{\omega}_{N-1}\left(T, \rho, Y_{1}, Y_{2}, \ldots, Y_{N-1}\right)}{\rho}
\end{array}\right),
$$

where $\rho$ is a constant. Note that we have replaced the independent variable $p$ in Eq. (29) with $\rho$, as is explained in [8]. We solve this system of stiff ODEs with a numerical package [10]. For the full details on numerical implementation, see [7].

It is important to note that $T$ is a function of the mass fractions when solving Eq. (35). A proper procedure for evaluating this function for temperature is described in subsection 3.2. However, as long as we follow the procedure dictated by the time splitting, there is no ambiguity about how to properly treat the temperature during the chemical reaction step. Contrary to common practices, temperature should not be frozen during this step, nor is there a need to derive an ODE with which to co-evolve the temperature. Instead, temperature is an implicit function of the mass fractions, as well as the other conserved variables that are held constant during this part of the splitting. All that is required is to properly evaluate the temperature as a function of these quantities, whenever a value is required. Since the functional relationship is implicit, this amounts to finding the root of a scalar equation for the temperature.

\subsection{Solving for Temperature}

It is necessary to compute the temperature from the conserved variables. We get an expression for the temperature by combining the energy equation with the equation of state. Combining Eq. (28) with Eq. (20) yields 


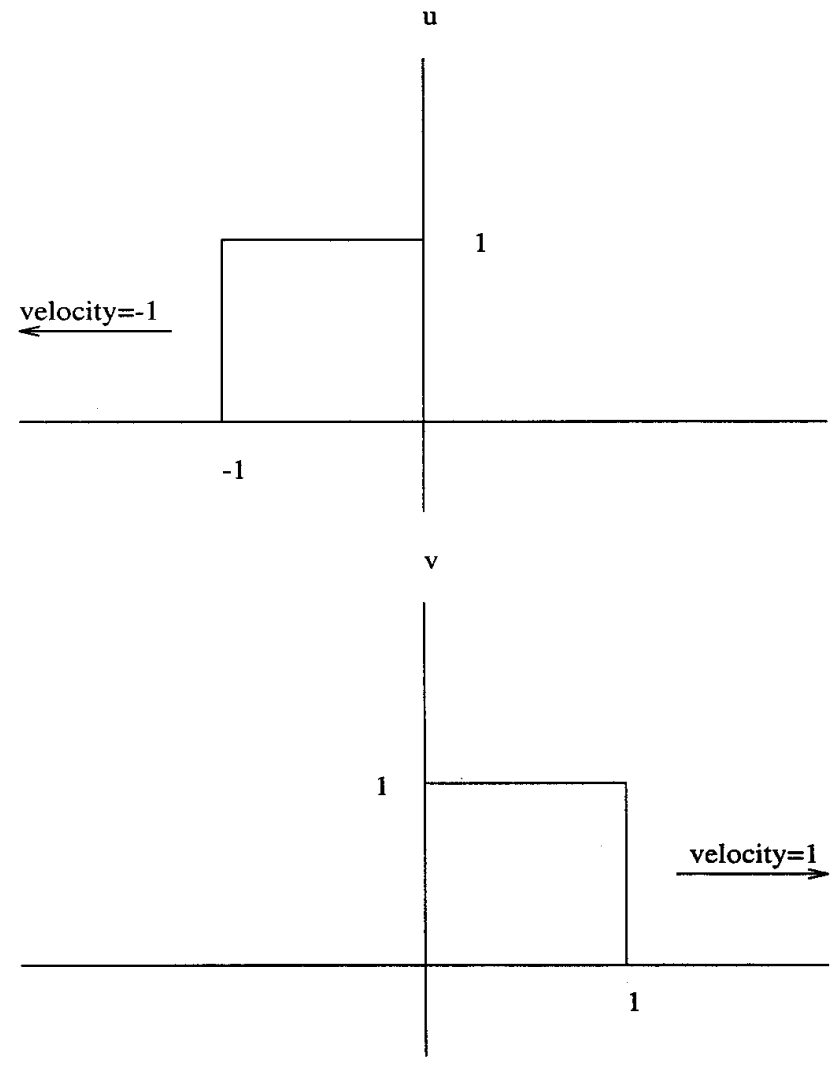

FIG. 1. Initial data for the two independent wave equations, resulting in simple translation to the left and to the right.

$$
\begin{aligned}
T & =\frac{-E+\rho\left(u^{2}+v^{2}\right) / 2+\rho h(T)}{\rho\left(\sum_{i=1}^{N} Y_{i} R_{i}\right)} \\
& =C_{1}+C_{2} h(T),
\end{aligned}
$$

where $C_{1}$ and $C_{2}$ are constants if the conserved variables are fixed. Note that $h(T)$ is defined in Eq. (14).

If we have a calorically perfect gas, then Eq. (36) can be written in the form,

$$
T=\frac{C_{3}}{1-C_{4} c_{p}},
$$

where $C_{3}$ and $C_{4}$ are constants if the conserved variables are fixed. In this case we have an explicit equation for the temperature.

However, if we have a thermally perfect gas, Eq. (36) is implicit for the temperature. We rewrite Eq. (36) as

$$
f(T)=T-C_{1}-C_{2} h(T)=0
$$

for a thermally perfect gas. Note that

$$
\frac{d f(T)}{d T}=1-C_{2} \frac{d h(T)}{d T}=1-C_{2} c_{p}(T)=1-\frac{c_{p}(T)}{R}=\frac{-1}{\gamma-1},
$$

where $\gamma$ is a function of temperature. Since $\gamma$ is always greater than one, this shows that $f(T)$ is a strictly decreasing function of temperature.

We can solve Eq. (38) with the Newton-Raphson iteration [3] applied to the temperature. The iteration is of the form

$$
T_{n+1}=T_{n}-f\left(T_{n}\right)\left[\frac{T_{n}-T_{n-1}}{f\left(T_{n}\right)-f\left(T_{n-1}\right)}\right],
$$

where the temperature from the last time step is used for $T_{0}$ and we set $T_{-1}=T_{0}+1$. Since the Newton-Raphson iteration is not guaranteed to converge, it is better to use it for only a few iterations. We use it for 5 iterations, then switch to bisection [3] if we are not within an acceptable error tolerance. In practice, Newton-Raphson has always converged in at most 5 iterations.

In order to do the above iteration for the temperature, we need to be able to calculate the enthalpy $h(T)$. To obtain a convenient form, integrate Eq. (10) starting from $T=298 \mathrm{~K}$ to get

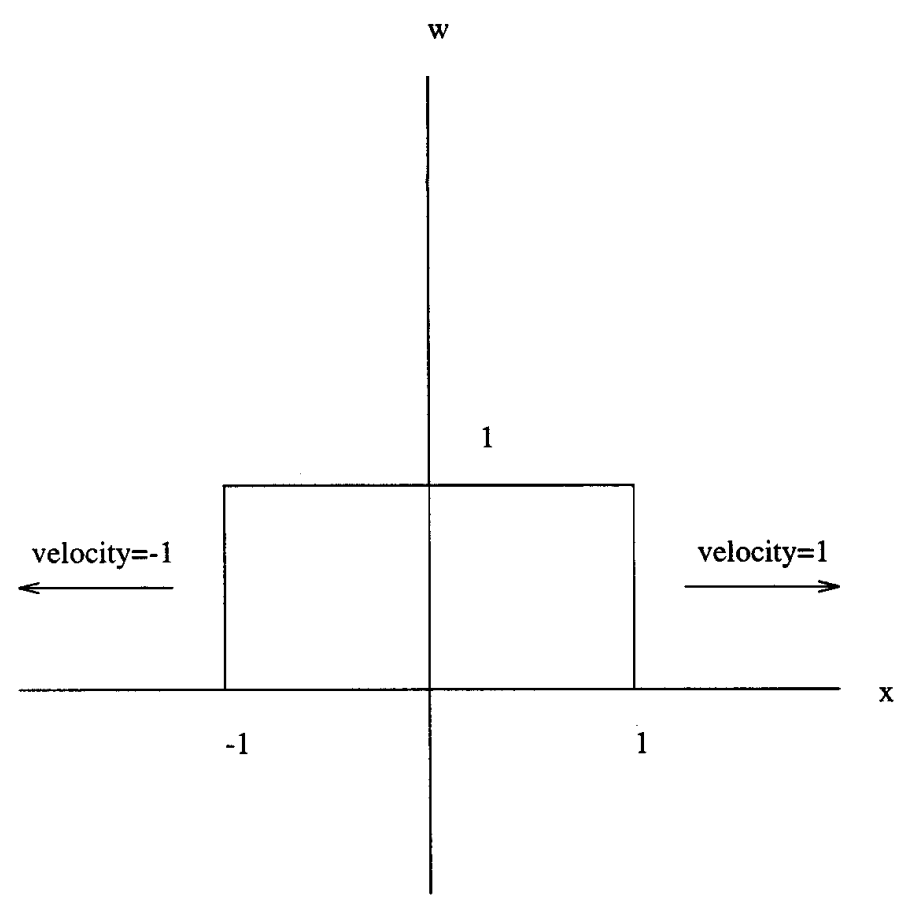

FIG. 2. Initial data for the separating box problem, as seen in one of the mixed fields. The evolution will split this initial box into two separate boxes traveling in opposite directions. 


$$
h_{i}(T)=h_{i}^{298}+\int_{298}^{T} c_{p i}(s) d s
$$

where $h_{i}^{298}$ is the enthalpy per unit mass at $298 \mathrm{~K}$ for species $i$. This is also sometimes called the heat of formation at $298 \mathrm{~K}$, which is a standard constant that can be found in the JANAF Thermochemical Tables [13]. If we assume that we have a calorically perfect gas, then we could use $298 \mathrm{~K}$ to evaluate our constant value for $c_{p i}$, defining this notationally as $c_{p i}^{298}$. Then Eq. (41) becomes

$$
h_{i}(T)=h_{i}^{298}+c_{p i}^{298}(T-298)
$$

for a calorically perfect gas with reference temperature of 298K.

To speed up the actual implementation, we construct a table of $h_{i}(T)$ for each species including every integer temperature between $298 \mathrm{~K}$ and $4800 \mathrm{~K}$. We approximate the integral to desired accuracy, using CHEMKIN to give us the values of $c_{p i}(T)$ when needed. This is done once at the beginning of the code. During computation, if we need $h_{i}(T)$ for a non-integral value of the temperature, we interpolate linearly.

Simple modification of this table for $h_{i}(T)$ enables calculations of calorically perfect mixtures. One could also have certain gases be thermally perfect with others calorically perfect. Further, a single gas could be thermally perfect in one temperature range and calorically perfect in another temperature range.

\section{EIGENVALUES AND EIGENVECTORS}

Many modern numerical methods for compressible gas flows require complete characteristic data-i.e., the eigensystem of the linearized convective flux matrix-as an essential part of the numerical discretization. For practical calculations, analytical expressions are required for the eigensystem, rather than general but costly iterative numerical procedures.

However, for equations describing the flow of many interacting species, the convection terms for momentum and energy can be far from simple, due to the complicated equation of state. Finding the Jacobian matrix of the convective flux with respect to the conserved variables can be a tedious calculation, and solving analytically for the corresponding eigensystem may seem impossible. Thus, it is tempting to try and simplify these calculations by dropping small terms or treating non-constant terms as approximately constant. But this practice can lead to unexpected numerical difficulties.

The nature of these difficulties can be understood as follows. If the eigensystem is slightly perturbed, the corresponding characteristic fields are mixed. Consider
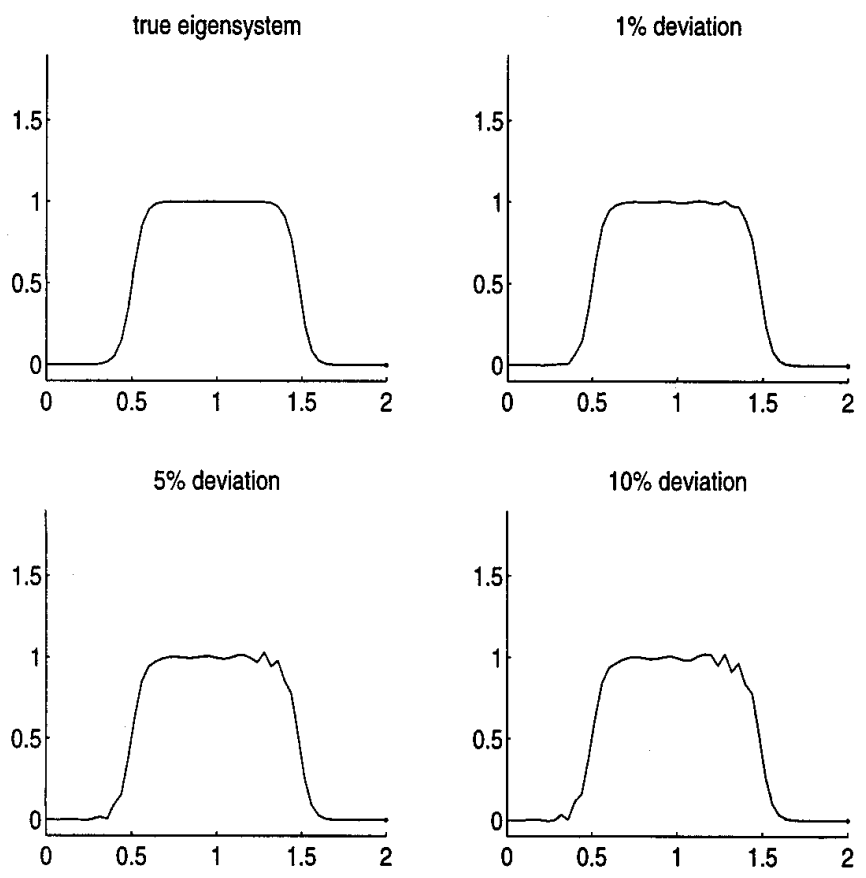

FIG. 3. Numerical solutions for the separating box problem, showing the oscillations that result from using an approximate eigensystem, deviating from the true eigensystem by the amounts shown. The results for the box to the right are shown, while the box to the left has a symmetric result.

a positive eigenvalue field which has been incorrectly mixed with a negative eigenvalue field. One-sided upwind differencing on this mixed field will result in one-sided downwind differencing on the field which is incorrectly represented by the eigenvalue. This will result in fields that have their convection discretized as a linear combination of upwind and downwind differencing. Even though the downwind portion may be "small," it can still contribute a significant oscillatory error near discontinuities (shocks or contacts).

\subsection{Example: Separating Box Problem}

Consider the following two one-way wave equations and their respective solutions,

$$
\begin{array}{ll}
u_{t}+u_{x}=0, & u(x, t)=u_{0}(x-t) \\
v_{t}-v_{x}=0, & v(x, t)=v_{0}(x+t),
\end{array}
$$

where the initial data $u_{0}$ and $v_{0}$ are given in Fig. 1 . The solutions to these equations move left and right as shown by the arrows in Fig. 1. Now consider changing the variables by letting $w=v+u$ and $z=v-u$. This yields a system of differential equations 

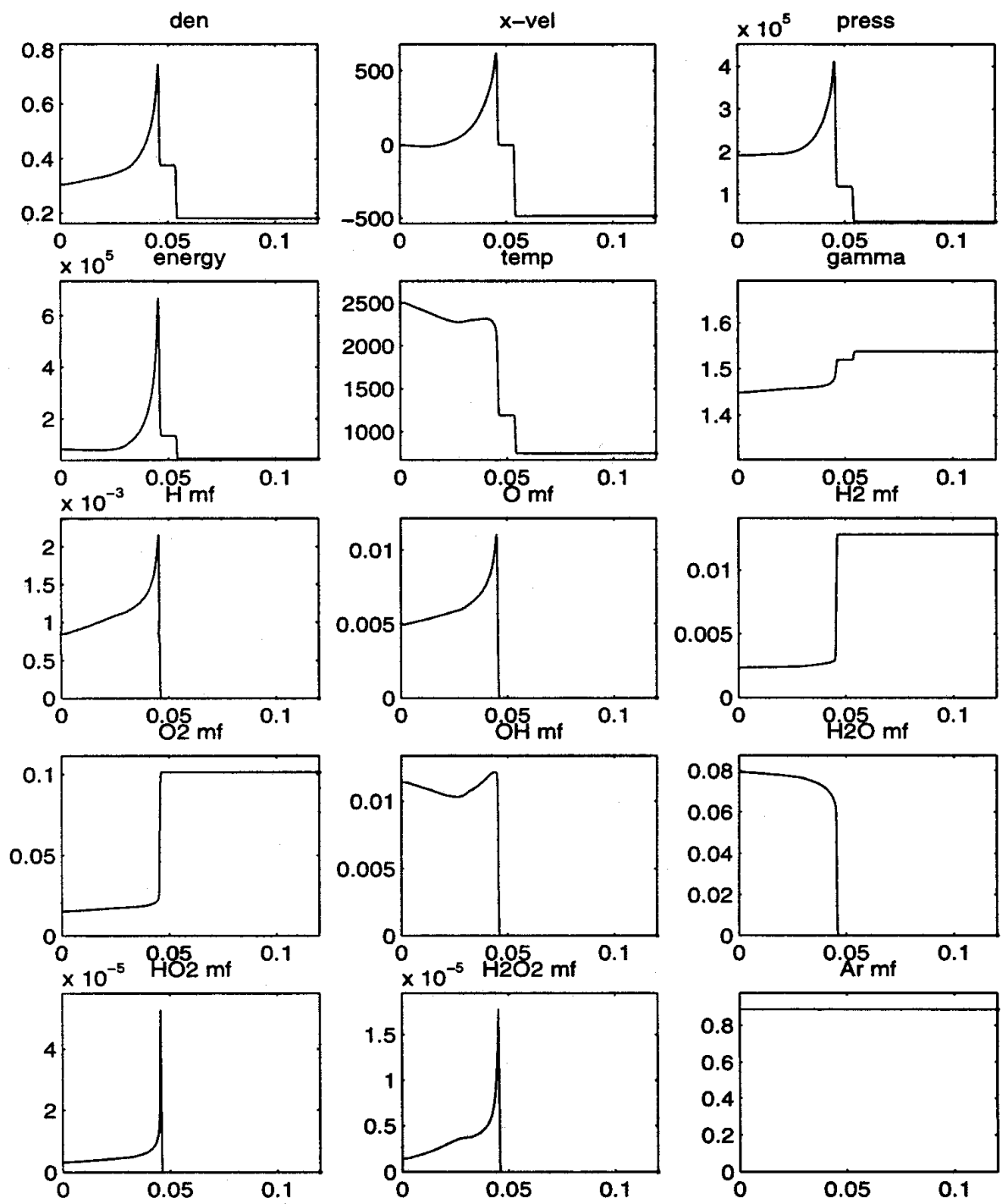

FIG. 4. Thermally Perfect Solution (1900 steps). The reaction wave and the shock are traveling from left to right. The shock is still in the lead, but losing ground.

$$
\left(\begin{array}{l}
w \\
z
\end{array}\right)_{t}+\left(\begin{array}{c}
z \\
w
\end{array}\right)_{x}=0
$$

with initial data $w_{0}=v_{0}+u_{0}$ and $z_{0}=v_{0}-u_{0}$. Also note that the solution of this system is

$$
\begin{aligned}
& w(x, t)=v_{0}(x-t)+u_{0}(x+t) \\
& z(x, t)=v_{0}(x-t)-u_{0}(x+t)
\end{aligned}
$$

which is obvious from the change of variables. Figure 2 shows the initial data for $w$ which consists of two open unit boxes defined on $(-1,0) \cup(0,1)$. As time evolves the boxes travel in opposite directions, as depicted by the arrows in Fig. 2.
Consider the following Jacobian matrix and associated eigensystem

$$
\begin{aligned}
J & =\left(\begin{array}{cc}
0 & (1-\varepsilon)^{2} \\
(1+\varepsilon)^{2} & 0
\end{array}\right) \\
\lambda^{1} & =-1+\varepsilon^{2}, \quad \lambda^{2}=1-\varepsilon^{2} \\
\mathbf{L}^{1} & =\left(\frac{1+\varepsilon}{2}, \frac{-1+\varepsilon}{2}\right), \quad \mathbf{L}^{2}=\left(\frac{1+\varepsilon}{2}, \frac{1-\varepsilon}{2}\right) \\
\mathbf{R}^{1} & =\left(\begin{array}{c}
\frac{1}{1+\varepsilon} \\
\frac{-1}{1-\varepsilon}
\end{array}\right), \quad \mathbf{R}^{2}=\left(\begin{array}{c}
\frac{1}{1+\varepsilon} \\
\frac{1}{1-\varepsilon}
\end{array}\right) .
\end{aligned}
$$



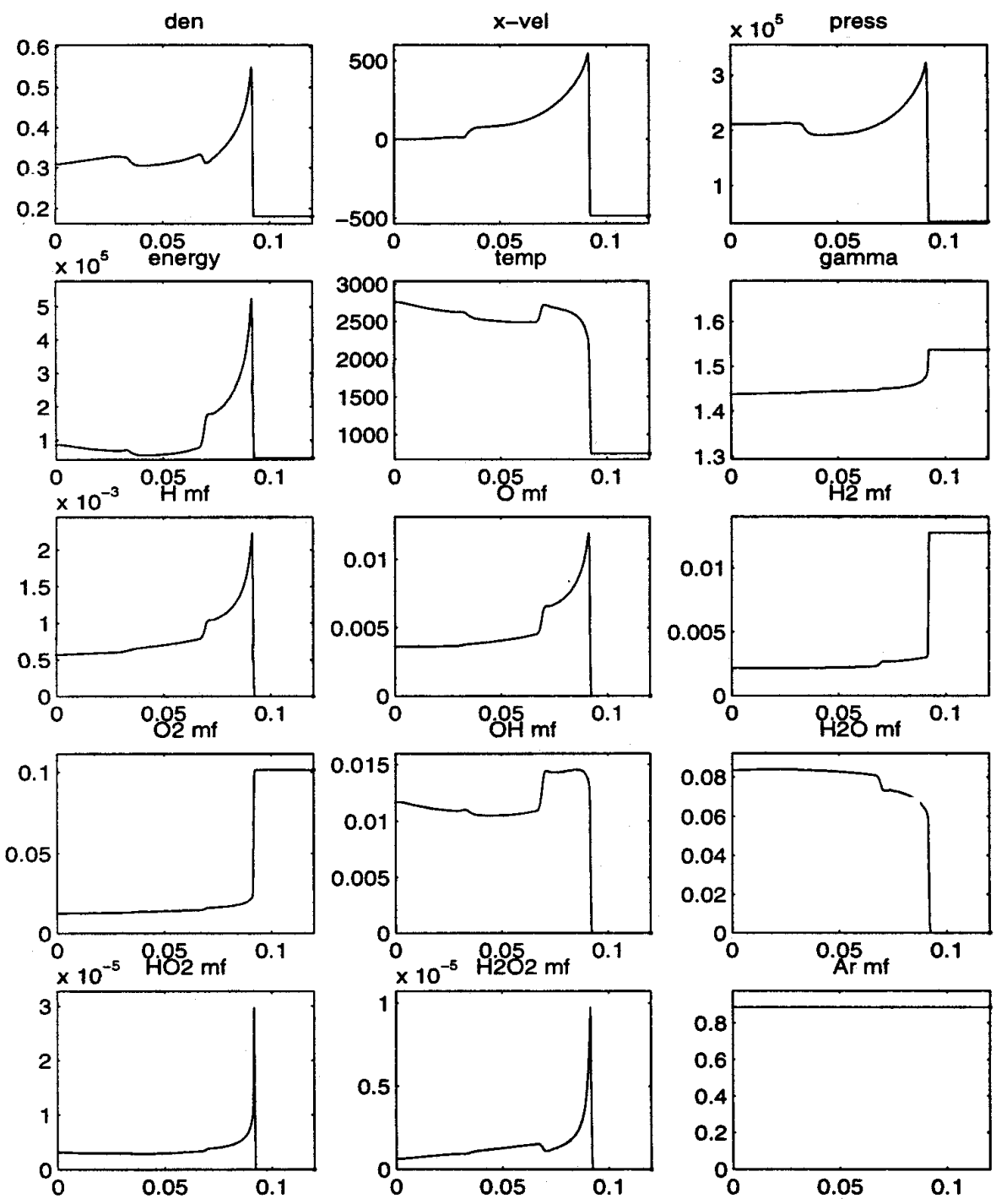

FIG. 5. Thermally Perfect Solution (2300 steps). The reaction wave has overtaken the shock, with the result splitting into three distinct waves. From left to right: a rarefaction wave, a contact discontinuity, and a detonation wave.

If we set $\varepsilon=0$, then this is the Jacobian and eigensystem for the convection term in Eq. (45). Otherwise, a nonzero $\varepsilon$ gives a perturbation of the Jacobian matrix. This yields a different eigensystem, and is designed so as to mimic ignoring small terms when computing complicated Jacobians.

We will now solve Eq. (45) numerically with 3rd order ENO on the convection terms, and 3rd order TVD RungeKutta in time. We set $\varepsilon=0$, which yields the true eigensystem. Figure 3 shows the results for the box moving to the right. Results for the box moving to the left are symmetric. We also solve with $\varepsilon^{2}=0.01, \varepsilon^{2}=0.05$, and $\varepsilon^{2}=0.1$. These give a $1 \%, 5 \%$, and a $10 \%$ perturbation of the eigenvalues, respectively. Again Fig. 3 shows the results for the box on the right. The box on the left has symmetric results.
One can see that ENO and TVD Runge-Kutta admit significant oscillations even on small perturbations of the Jacobian matrix. It is therefore not advisable to alter a Jacobian matrix in order to simplify the computation of an eigensystem.

\subsection{2-D Euler with Multiple Species}

Consider the convective part of the conservation equations (1), (2), and (3). This is a system of $N+3$ equations, so there will be $N+3$ eigenvalues with associated eigenvectors. For the eigenvalues and eigenvectors of the Jacobian matrix of $\mathbf{F}(\mathbf{U})$ in Eq. (2), set $A=1$ and $B=0$ below. For the eigenvalues and eigenvectors of the Jacobian matrix of $\mathbf{G}(\mathbf{U})$ in Eq. (2), set $A=0$ and $B=1$ below. 

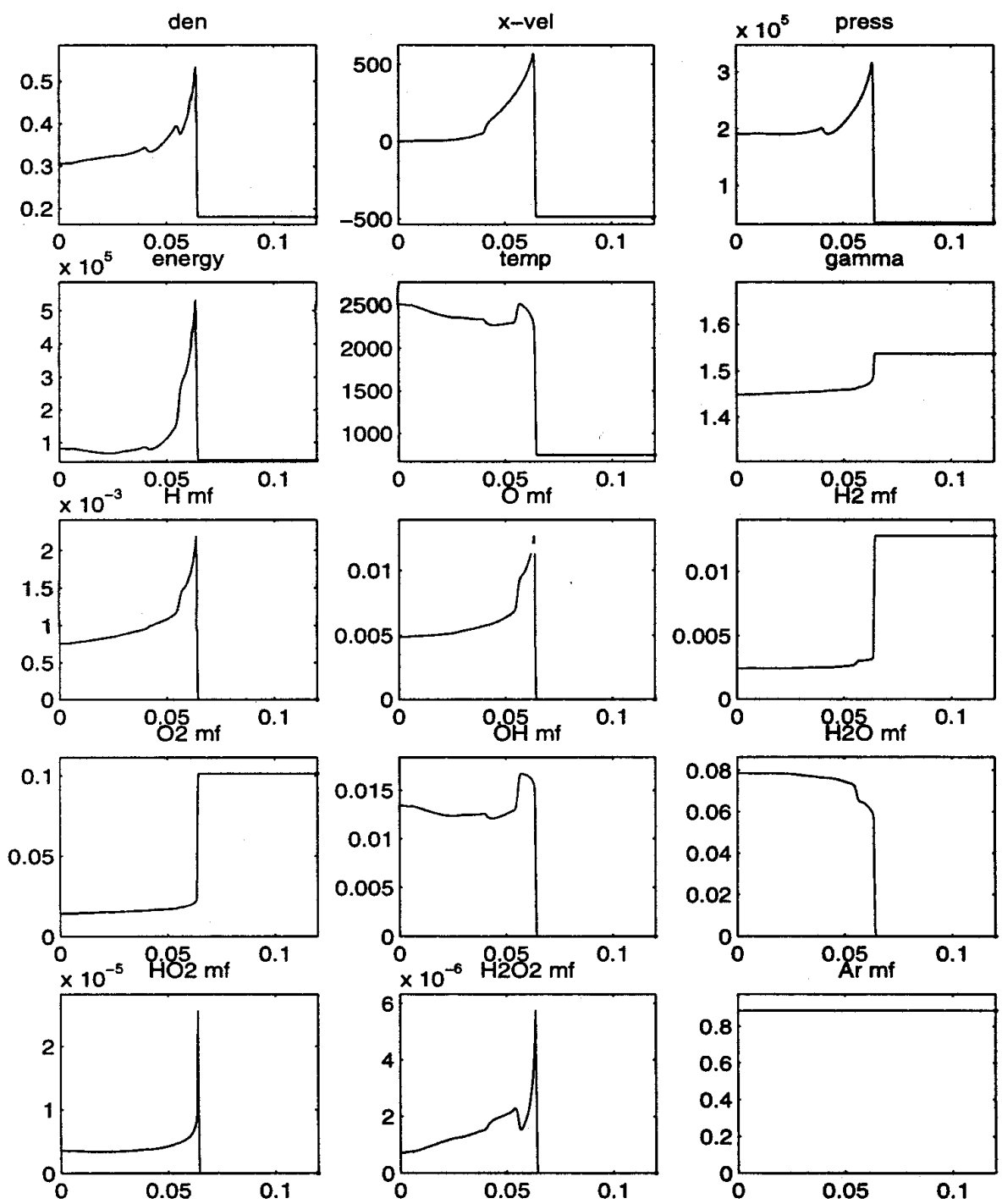

FIG. 6. Calorically Perfect Solution (1900 steps). The calorically perfect assumptions drive the reaction and cause the reaction wave to prematurely overtake the shock.

Based on Eq. (14) and (13), we can calculate the following derivatives of $\rho h$ with respect to the conserved variables,

$$
\begin{aligned}
& \frac{d(\rho h)}{d \rho}=h_{N}+\rho c_{p} \frac{d T}{d \rho} \\
& \frac{d(\rho h)}{d(\rho u)}=\rho c_{p} \frac{d T}{d(\rho u)} \\
& \frac{d(\rho h)}{d(\rho v)}=\rho c_{p} \frac{d T}{d(\rho v)} \\
& \frac{d(\rho h)}{d E}=\rho c_{p} \frac{d T}{d E}
\end{aligned}
$$

$$
\frac{d(\rho h)}{d\left(\rho Y_{i}\right)}=h_{i}-h_{N}+\rho c_{p} \frac{d T}{d\left(\rho Y_{i}\right)}
$$

where Eq. (56) holds for $i=1$ to $N-1$.

These derivatives of $\rho h$ are valid for both a mixture of thermally perfect gases and a mixture of calorically perfect gases. Further, they are valid for any mixture of gases in which $c_{p, i}$ is defined as a function of temperature for each species. One could construct a table of $c_{p, i}$ 's which obeys any combination of thermally perfect and calorically perfect assumptions. We use CHEMKIN [10] to compute realistic values for $c_{p, i}$. For some lower temperatures where CHEMKIN does not have data, we extrapolate using a calorically perfect assumption. 

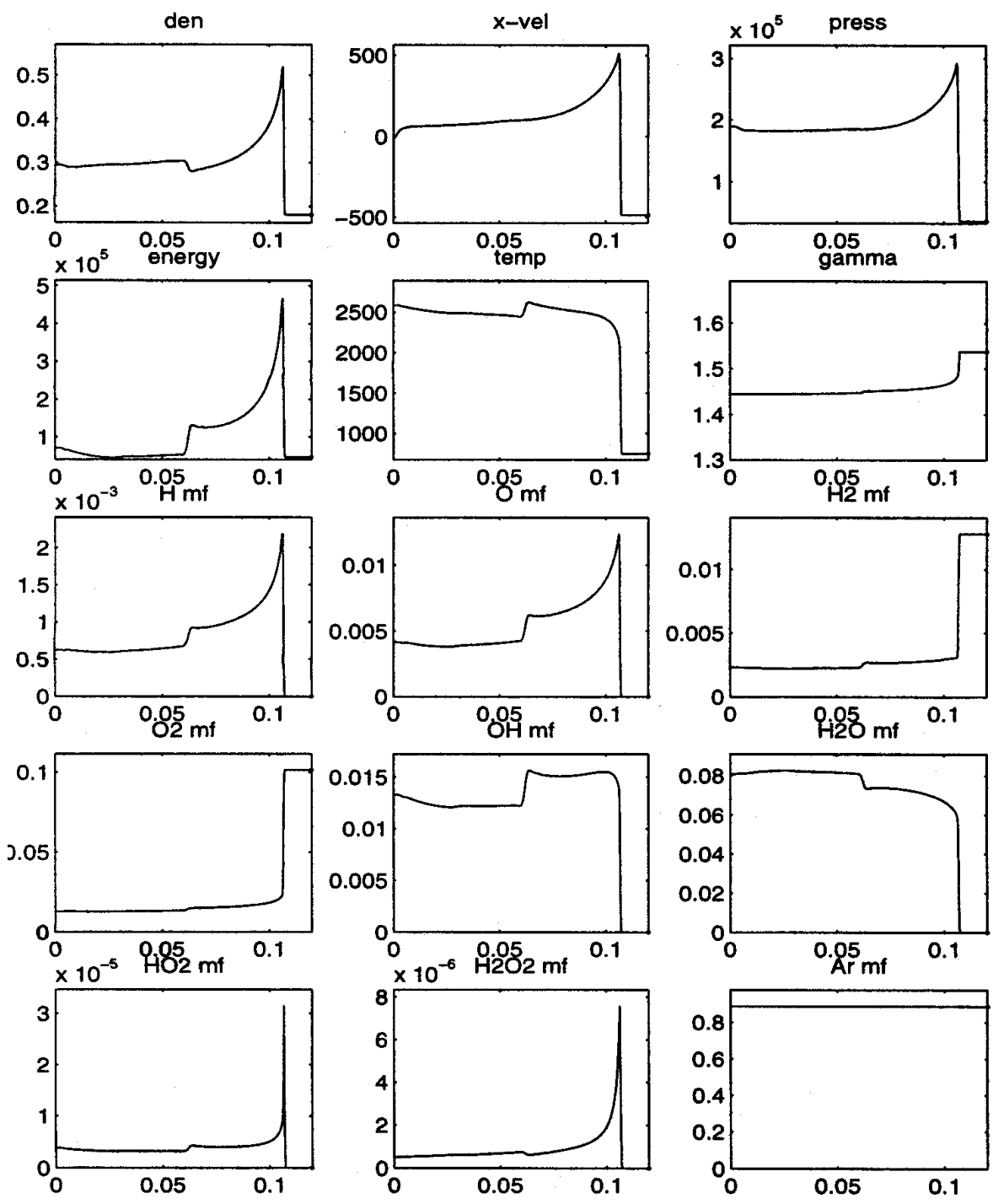

FIG. 7. Calorically Perfect Solution (2300 steps). The calorically perfect assumptions drive the reaction and force a non-physical accelerated evolution of the solution.

From Eq. (3) we can write

$$
p=-E+\frac{\rho\left(u^{2}+v^{2}\right)}{2}+\rho h
$$

and take derivatives with respect to the conserved variables to obtain

$$
\begin{aligned}
\frac{d p}{d \rho} & =\frac{-\left(u^{2}+v^{2}\right)}{2}+h_{N}+\rho c_{p} \frac{d T}{d \rho} \\
\frac{d p}{d(\rho u)} & =u+\rho c_{p} \frac{d T}{d(\rho u)} \\
\frac{d p}{d(\rho v)} & =v+\rho c_{p} \frac{d T}{d(\rho v)}
\end{aligned}
$$

$$
\begin{gathered}
\frac{d p}{d E}=-1+\rho c_{p} \frac{d T}{d E} \\
\frac{d p}{d\left(\rho Y_{i}\right)}=h_{i}-h_{N}+\rho c_{p} \frac{d T}{d\left(\rho Y_{i}\right)},
\end{gathered}
$$

where Eq. (62) holds for $i=1$ to $N-1$. Note that we have used Eqs. (52)-(56). Now take derivatives with respect to the conserved variables of Eq. (20) to obtain

$$
\begin{aligned}
\frac{d p}{d \rho} & =R_{N} T+\rho R \frac{d T}{d \rho} \\
\frac{d p}{d(\rho u)} & =\rho R \frac{d T}{d(\rho u)}
\end{aligned}
$$



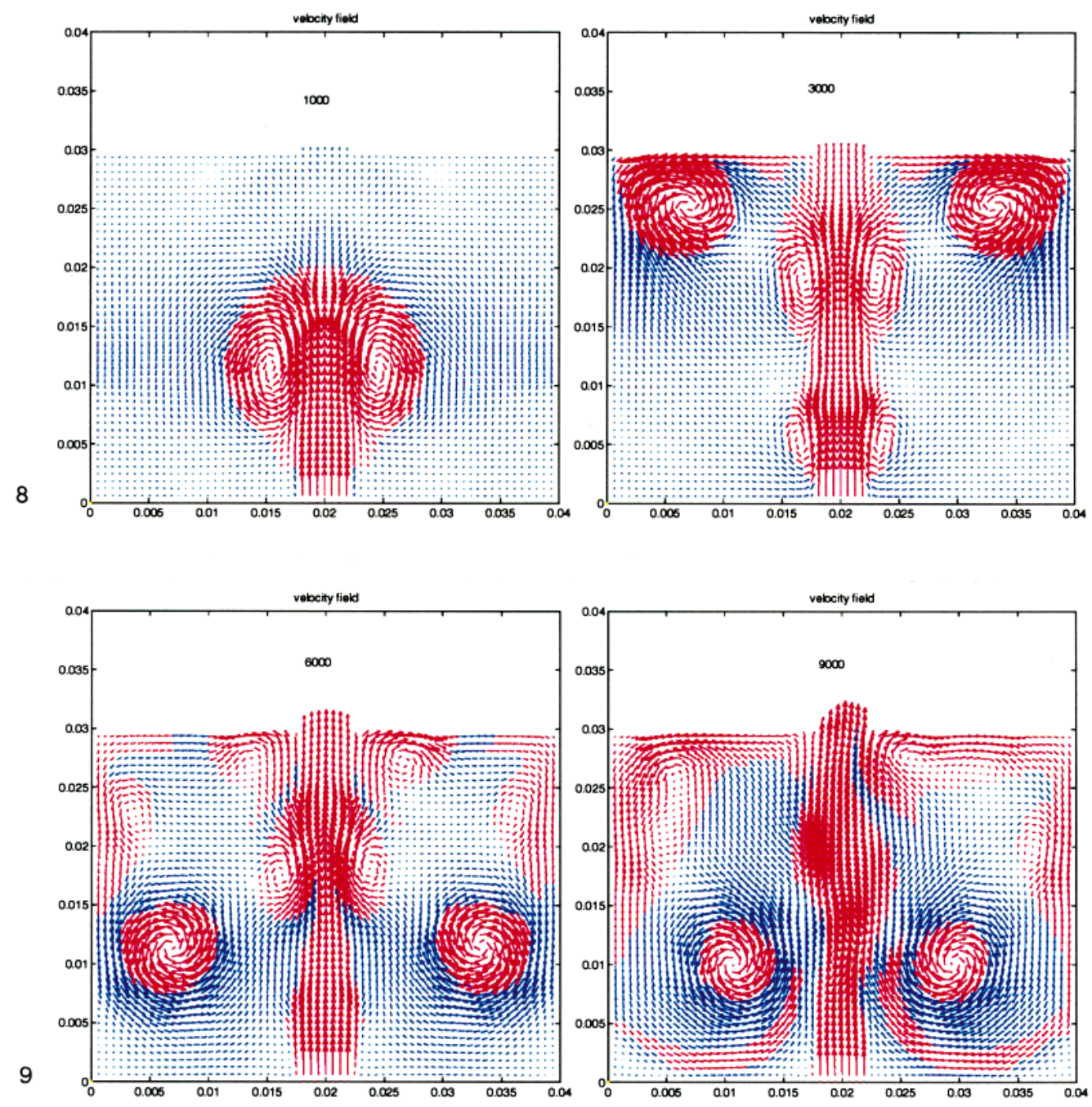

FIG. 8. Velocity field in the combustor problem at times 1000 and 3000 microseconds. The jet of $\mathrm{H}_{2}$ gas (red) is continuously injected into the $\mathrm{O}_{2}$ gas (blue) in the chamber, resulting in vortex shedding.

FIG. 9. Velocity field in the combustor problem at times 6000 and 9000 microseconds. The primary vortices develop, entrain $\mathrm{H}_{2}$ and $\mathrm{O}_{2}$, and enhance mixing.

$$
\begin{aligned}
\frac{d p}{d(\rho v)} & =\rho R \frac{d T}{d(\rho v)} \\
\frac{d p}{d E} & =\rho R \frac{d T}{d E} \\
\frac{d p}{d\left(\rho Y_{i}\right)} & =\left(R_{i}-R_{N}\right) T+\rho R \frac{d T}{d\left(\rho Y_{i}\right)}
\end{aligned}
$$

which we can use to eliminate the derivatives of $T$ in Eqs. (58)-(62). We can than solve for the derivatives of $p$ to obtain

$$
\frac{d p}{d \rho}=(\gamma-1)\left(\frac{u^{2}+v^{2}}{2}-h_{N}+\frac{c_{p} R_{N} T}{R}\right)
$$

$$
\begin{aligned}
& \frac{d p}{d(\rho u)}=(\gamma-1)(-u) \\
& \frac{d p}{d(\rho v)}=(\gamma-1)(-v) \\
& \frac{d p}{d E}=(\gamma-1) \\
& \frac{d p}{d\left(\rho Y_{i}\right)}=(\gamma-1)\left(-h_{i}+h_{N}+\frac{c_{p}\left(R_{i}-R_{N}\right) T}{R}\right)
\end{aligned}
$$

which we will need below. 

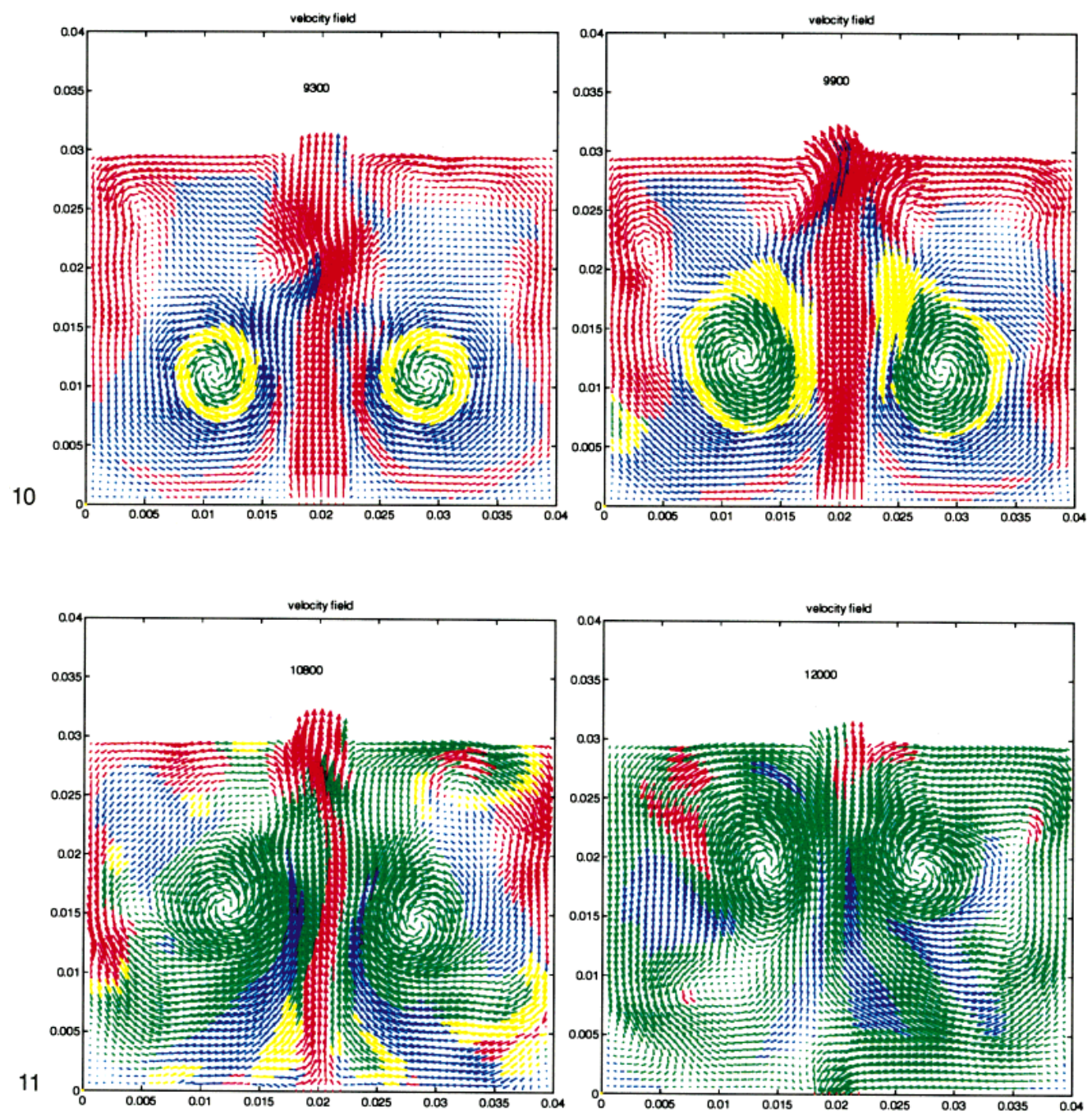

FIG. 10. Velocity field in the combustor problem at times 9300 and 9900 microseconds. The mixture ignites in two primary vortices and a combustion front, indicated by the presence of $\mathrm{OH}$ (yellow), spreads outward, leaving behind the combustion product $\mathrm{H}_{2} \mathrm{O}$ (green).

FIG. 11. Velocity field in the combustor problem at times 10800 and 12000 microseconds. There is widespread burning, with much of the $\mathrm{O}_{2}$ consumed in the process.

The Jacobian matrix can be written as

$$
\hat{u} I+J F+J B
$$

with

$$
\begin{aligned}
J F= & \left(\frac{d p}{d \rho} \mathbf{J}_{f} \frac{d p}{d(\rho u)} \mathbf{J}_{f} \frac{d p}{d(\rho v)} \mathbf{J}_{f} \frac{d p}{d E} \mathbf{J}_{f}\right. \\
& \left.\frac{d p}{d\left(\rho Y_{1}\right)} \mathbf{J}_{f} \cdots \frac{d p}{d\left(\rho Y_{N-1}\right)} \mathbf{J}_{f}\right) \\
J B= & \left(\begin{array}{lllll}
1 \mathbf{J}_{b} & u \mathbf{J}_{b} & v \mathbf{J}_{b} & H \mathbf{J}_{b} & \left.Y_{1} \mathbf{J}_{b} \cdots Y_{N-1} \mathbf{J}_{b}\right)^{T},
\end{array}\right.
\end{aligned}
$$

where $I$ is the $N+3$ by $N+3$ identity matrix, and

$$
\mathbf{J}_{f}=\left(\begin{array}{c}
0 \\
A \\
B \\
\hat{u} \\
0 \\
\vdots \\
0
\end{array}\right), \quad \mathbf{J}_{b}=\left(\begin{array}{c}
-\hat{u} \\
A \\
B \\
0 \\
0 \\
\vdots \\
0
\end{array}\right) .
$$


The eigenvalues of this Jacobian matrix are

$$
\begin{aligned}
\lambda^{1} & =\hat{u}-c \\
\lambda^{2} & =\cdots=\lambda^{N+2}=\hat{u} \\
\lambda^{N+3} & =\hat{u}+c .
\end{aligned}
$$

The left eigenvectors, $\mathbf{L}^{p}$, are the rows of the matrix and reflecting off. After a delay a reaction wave kicks in at the boundary. This reaction wave picks up steam and merges with the shock causing a split into 3 waves. From wall to outflow (left to right) these waves are a rarefaction, a contact discontinuity, and a detonation wave.

We apply the 1-D Euler equations for multi-species flow with chemical reactions. Assume that we have a 2/1/7 molar

$$
\left(\begin{array}{ccccccc}
\frac{b_{2}}{2}+\frac{\hat{u}}{2 c}+\frac{b_{3}}{2} & -\frac{b_{1} u}{2}-\frac{A}{2 c} & -\frac{b_{1} v}{2}-\frac{B}{2 c} & \frac{b_{1}}{2} & \frac{-b_{1} z_{1}}{2} & \ldots & \frac{-b_{1} z_{N-1}}{2} \\
1-b_{2}-b_{3} & b_{1} u & b_{1} v & -b_{1} & b_{1} z_{1} & \ldots & b_{1} z_{N-1} \\
\hat{v} & B & -A & 0 & 0 & \ldots & 0 \\
-Y_{1} & 0 & 0 & 0 & & & \\
\vdots & \vdots & \vdots & \vdots & & I & \\
-Y_{N-1} & 0 & 0 & 0 & & & \\
\frac{b_{2}}{2}-\frac{\hat{u}}{2 c}+\frac{b_{3}}{2} & -\frac{b_{1} u}{2}+\frac{A}{2 c} & -\frac{b_{1} v}{2}+\frac{B}{2 c} & \frac{b_{1}}{2} & \frac{-b_{1} z_{1}}{2} & \ldots & \frac{-b_{1} z_{N-1}}{2}
\end{array}\right) .
$$

The right eigenvectors, $\mathbf{R}^{p}$, are the columns of the matrix

$$
\left(\begin{array}{ccccccc}
1 & 1 & 0 & 0 & \ldots & 0 & 1 \\
u-A c & u & B & 0 & \ldots & 0 & u+A c \\
v-B c & v & -A & 0 & \ldots & 0 & v+B c \\
H-\hat{u} c & H-\frac{1}{b_{1}} & -\hat{v} & z_{1} & \ldots & z_{N-1} & H+\hat{u} c \\
Y_{1} & Y_{1} & 0 & & & & Y_{1} \\
\vdots & \vdots & \vdots & & I & & \vdots \\
Y_{N-1} & Y_{N-1} & 0 & & & & Y_{N-1}
\end{array}\right) .
$$

Here $I$ is the $N-1$ by $N-1$ identity matrix, and

$$
\begin{gathered}
q^{2}=u^{2}+v^{2}, \quad \hat{u}=A u+B v, \quad \hat{v}=A v-B u \\
c=\sqrt{\frac{\gamma p}{\rho}} \\
b_{1}=\frac{\gamma-1}{c^{2}}, \quad b_{2}=1+b_{1} q^{2}-b_{1} H \\
b_{3}=b_{1} \sum_{i=1}^{N-1} Y_{i} z_{i}, \quad z_{i}=\frac{-1}{\gamma-1}\left(\frac{d p}{d\left(\rho Y_{i}\right)}\right) .
\end{gathered}
$$

\section{NUMERICAL EXAMPLES}

\subsection{1-D Chemically Reacting "SOD" Shock Tube}

We consider a one-dimensional test problem with chemical reactions. We have a shock hitting a solid wall boundary ratio of $\mathrm{H}_{2} / \mathrm{O}_{2} /$ Ar. All gases involved are assumed to be thermally perfect. We use a full chemical mechanism, see [8] for details.

We use the initial data

$$
\begin{aligned}
& \rho=.072 \frac{\mathrm{kg}}{\mathrm{m}^{3}}, \quad u=0 \frac{\mathrm{m}}{\mathrm{s}} \\
& p=7173 \frac{\mathrm{J}}{\mathrm{m}^{3}}
\end{aligned}
$$

on the left side of the shock. Then we use a numerical algorithm [7] to calculate conditions on the right side which are consistent with the Rankine-Hugoniot equations for a shock. This yields initial data of

$$
\begin{aligned}
& \rho=.18075 \frac{\mathrm{kg}}{\mathrm{m}^{3}}, \quad u=-487.34 \frac{\mathrm{m}}{\mathrm{s}} \\
& p=35594 \frac{\mathrm{J}}{\mathrm{m}^{3}}
\end{aligned}
$$

on the right side of the shock. We use a 12-cm domain for a time of $230 \mu \mathrm{s}, 400$ uniform grid cells, and 2300 equal time steps. A reflective boundary condition at the wall is implemented by adding ghost cells.

We will examine the solution after a total of 1900 time steps, and after 2300 time steps. The results are shown in Figs. 4 and 5. Next, we run the code under the calorically perfect assumptions. We use a reference temperature of 
298 K. The results are shown in Figs. 6 and 7. The calorically perfect assumptions drive the reaction, and cause a major difference in evolution of the solution.

\subsection{2-D Combustor Simulation}

Consider the 2-D Navier Stokes equations for multispecies flow with chemical reactions. All gases involved are assumed to be thermally perfect. See [8] for details on the chemical mechanism.

We have a $4 \mathrm{~cm}$ by $3 \mathrm{~cm}$ domain, with 64 by 48 cells. The time step taken was $10 \mu \mathrm{s}$. The initial data were a motionless mixture with $(T, p)=(700 \mathrm{~K}, 36100 \mathrm{~Pa})$. The mixture consists of a $2 / 7$ molar ratio of $\mathrm{O}_{2} / \mathrm{Ar}$ gas. There is an inflow of size $0.4375 \mathrm{~cm}$ at the bottom and an outflow of equal size at the top. At the inflow, we inject a 4/7 molar ratio of $\mathrm{H}_{2} / \mathrm{Ar}$, at $10 \mathrm{~m} / \mathrm{sec}$ with $(T, p)=(1166 \mathrm{~K}$, $121000 \mathrm{~Pa})$.

The results for the velocity field are shown in Figs. 8-11. The vectors are color coded to better illustrate the solution. Red arrows are for regions of the flow which have a high enough concentration of $\mathrm{H}_{2}$ gas to be considered fuel. Blue arrows are for regions which have enough $\mathrm{O}_{2}$ gas to dilute the fuel for combustion. Yellow arrows are for combustion regions. We use $\mathrm{OH}$ as the detector gas, as is common in actual engineering experiments. Green arrows are for regions of the flow which have undergone near complete reaction. These regions are composed of "waste" materials, primarily water vapor. The color coding is adjusted using thresholds which give one the idea of the underlying chemistry. A given cell may contain up to 9 gases.

Figure 8 (left) shows the initial injection of the hydrogen gas. The first set of vortices split to the right and left in Fig. 8 (right), after impact with the top of the container. They then continue in a circular type path as can be seen in Fig. 9 (left). In Fig. 9 (right), one can see that the smaller vortices are being destroyed by the global flow. In particular, note that the second pair of vortices is now a "half moon" shape at the bottom of the page.

Figure 10 (right and left) shows the ignition of the main vortices. The ignition quickly spreads throughout the container as can be seen in Fig. 11 (left). The amount of fuel and oxygen which was wasted, not part of the main reaction, can be seen in Fig. 11 (right).

\section{CONCLUSION}

We have presented an enthalpy-based formulation of the equations for multi-species compressible chemically reacting flow that is particularly well suited to numerical modeling with modern high accuracy methods.
We have shown how to properly time split these equations in order to efficiently integrate the stiff reaction terms while avoiding inaccurate or unnecessary common practices such as freezing the temperature, or introducing a temperature ODE.

We have derived new, simple expressions for the characteristics of the convective portion of the equations, which allow the application of many modern characteristic-based numerical methods.

We have used these equations, time splitting, and characteristics together with the finite difference ENO discretization to perform high accuracy calculations of representative 1-D and 2-D problems.

The framework and numerical results presented here show that the modern high accuracy numerical methods developed for gas dynamics can be usefully extended to the much more complicated problem of chemically reacting gas flows, and that these methods can effectively capture the complex combustion phenomena present in these flows.

\section{ACKNOWLEDGMENTS}

We thank Ann Karagozian, Bjorn Engquist, Chris Anderson, Owen Smith, and Dave Chopp for helpful comments, insights, and criticisms during the course of this work.

\section{REFERENCES}

1. J. D. Anderson, Hypersonic and High Temperature Gas Dynamics (McGraw-Hill, New York, 1989).

2. J. D. Anderson, Modern Compressible Flow (McGraw-Hill, New York, 1990).

3. K. E. Atkinson, An Introduction to Numerical Analysis (Wiley, New York, 1989).

4. W. Don and C. Quillen, Numerical simulation of shock-cylinder interactions. Part I. Resolution, preprint, 1994.

5. B. Engquist and B. Sjogren, Numerical approximation to hyperbolic conservation laws with stiff terms, UCLA CAM Report 89-07, March 1989 (unpublished).

6. B. Engquist and B. Sjogren, Robust difference approximations to stiff inviscid detonation waves, UCLA CAM Report 91-03, March 1991 (unpublished).

7. R. P. Fedkiw, A Survey of Chemically Reacting, Compressible Flows, Dissertation, UCLA, 1996.

8. R. Fedkiw, B. Merriman, and S. Osher, Numerical methods for a mixture of thermally perfect and/or calorically perfect gaseous species with chemical reactions, UCLA CAM Report 96-1, January 1996 (unpublished); http://www.math.ucla.edu/applied/cam/index.html.

9. E. Katzer and S. Osher, Efficient implementation of essentially nonoscillatory schemes for systems of nonlinear hyperbolic differential equations, UCLA CAM Report 88-14, May 1988 (unpublished).

10. M. Kee and Jefferson, CHEMKIN: A general purpose problem independent, transportable Fortran chemical kinetics code package, SAND 80-8003, Sandia National Laboratories, March 1986 (unpublished). 
11. E. S. Oran and J. P. Boris, Numerical Simulation of Reactive Flow (Elsevier, Amsterdam/New York, 1987).

12. C. W. Shu and S. Osher, Efficient implementation of essentially nonoscillatory shock capturing schemes, J. Comput. Phys. 83, 32-78 (1989).

13. D. R. Stall and H. Prophet, JANAF Thermochemical Tables (National Standard Reference Data Series, 1971).
14. J. C. Strikwerda, Finite Difference Schemes and Partial Differential Equations (Wadsworth, Belmont, CA, 1989).

15. V. Ton, A Numerical Method for Mixing/Chemically Reacting Compressible Flow with Finite Rate Chemistry, Dissertation, UCLA, 1993.

16. V. Ton, A. Karagozian, B. Engquist, and S. Osher, Numerical simulation of inviscid detonation waves, UCLA CAM Report 91-22, October 1991 (Theor. Comput. Fluid Dyn. 6, 161-179 (1994). 